\title{
Suppression of Grating Lobes in Stepped-Frequency Train
}

\author{
Irina Gladkova, Ph.D., City College of New York \\ Dmitry Chebanov, Ph.D., CUNY
}

Key Words: stepped-frequency train, autocorrelation function, grating lobes.

\begin{abstract}
For uniform frequency stepped pulse trains, there can be undesirable peaks of the autocorrelation function, known as "grating lobes". In this paper we address this issue, using an approach which allows us to suppress grating lobes below a desired threshold level in the case of appropriately chosen stepped frequency waveforms, i.e., sequences of narrowband pulses that span the desired bandwidth. We discuss in detail how to choose relevant parameters in order to produce such waveforms with small grating lobes, and give examples of waveforms with small overlap ratio. We also discuss the issue of high sidelobes in the vicinity of the main lobe, which are inevitable in a train of LFM waveforms, and show that it is possible to suppress these, as well as the grating lobes, by means of phase modulation.
\end{abstract}

\section{INTRODUCTION}

Frequency stepping is one of the known techniques employed by modern radars to achieve high range resolution. The main advantage of this approach is that the actual instantaneous bandwidth of a radar is quite small compared with the total processing bandwidth. This allows the transmission of waveforms with extremely wide overall bandwidth (and, as a consequence, the attainment of high range resolution) without the usage of the expensive hardware needed to support the wide instantaneous bandwidth.

A stepped-frequency waveform we consider in this paper consists of $N$ subpulses, each of duration $t_{p}$, separated in time by a repetition interval $t_{r}$ (for example, a stepped-frequency train consisting of LFM pulses is schematically depicted in Fig. 1). Each pulse has the same bandwidth $B$. A large total bandwidth is achieved by creating a constant step $\triangle f$ between the center frequencies of consecutive pulses.

It is well-known that the autocorrelation function of a stepped-frequency pulse train suffers from grating lobes that appear due to the presence of the constant frequency step $\triangle f$. These high spikes essentially reduce the waveform's resolution capabilities and, hence, they are undesirable. Recently, publications [1,2] have discussed different approaches leading to either acceptable suppression or complete elimination of the grating lobes. For instance, it was shown in [1] that a suitable choice of parameters at hand (i.e., $B, \triangle f$, and $t_{p}$ ) allows one to nullify several (or, sometimes, even all) grating lobes of an LFM pulse train. One of the conclusions that can be drawn from the relationship between $t_{p} B$ and $t_{p} \triangle f$ obtained in [1] is that the overlap ratio $B / \triangle f$ is large for large values of $t_{p} B$. Therefore, in order to increase the bandwidth significantly (under the restriction that the first two grating lobes are nullified), the number of pulses $N$ has to be large, i.e. the obtained relationship can only be applied to "Slow Burst" waveforms.

In this paper we suggest a modified method for suppressing grating lobes in stepped-frequency pulse trains. It can be considered as an extension of the technique introduced in [1] which allows "Quick Burst" waveforms to be considered. Our modification of the grating lobes suppression problem leads to a significantly different relationship between $t_{p} B$ and $t_{p} \triangle f$ which allows us to find waveforms with large $t_{p} B$, small ratio $B / \triangle f$, and small (rather then zero) grating lobes.

Another issue related to a stepped-frequency LFM pulse train is the high sidelobes of its autocorrelation function appearing in the close vicinity of the main lobe. It is known that within the chosen construction of the stepped-frequency waveform with constant time duration $t_{p}$, constant bandwidth $B$, constant step $\triangle f$, and small ratio $B / \triangle f$, it is not possible to suppress these few sidelobes. Nevertheless, as we show in section 4, if a linear FM pulse is replaced by a non-linear FM pulse, then it is possible to suppress the sidelobes near the main lobe as well as the grating lobes.

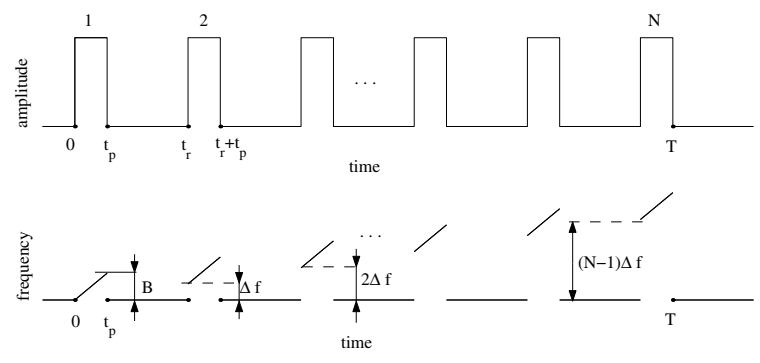

Figure 1: Stepped-frequency LFM pulse train.

\section{STEPPED-FREQUENCY PULSE TRAINS}

We start by considering a uniform pulse train

$$
u_{N}(t)=\frac{1}{\sqrt{N}} \sum_{n=0}^{N-1} u_{1}\left(t-n t_{r}\right)
$$


of $\mathrm{N}$ identical "simple" pulses of duration $t_{p}$ and bandwidth $B_{1}$

$$
u_{1}(t)=\frac{1}{\sqrt{t_{p}}} \operatorname{rect}\left(\frac{t}{t_{p}}\right) \exp (j \pi \phi(t))
$$

that are separated in time by $t_{r} \geq 2 t_{p}$. At this point, we do not set any restrictions on the choice of the phase $\phi(t)$ of pulse (2).

One of the known effective ways to increase the bandwidth of pulse train (1) is to add a frequency step $\triangle f$ between consecutive pulses. This can be done via multiplication of the entire train (1) by $\exp \left(j \pi k_{s} t^{2}\right.$ ) (we assume a positive frequency slope $k_{s}>0$ ), resulting in a stepped-frequency pulse train (see Fig. $1)$ :

$$
\begin{aligned}
& u_{s}(t)=\frac{1}{\sqrt{N}} \exp \left(j \pi k_{s} t^{2}\right) \sum_{n=0}^{N-1} u_{1}\left(t-n t_{r}\right) \\
& =\frac{1}{\sqrt{N}} \sum_{n=0}^{N-1} u\left(t-n t_{r}\right),
\end{aligned}
$$

where $u(t)=\frac{1}{\sqrt{t_{p}}} \operatorname{rect}\left(t / t_{p}\right) \exp \left(j \pi\left(\phi(t)+k_{s} t^{2}\right)\right)$ and $\triangle f=$ $k_{s} t_{r}$. We denote the ultimate bandwidth of each pulse in the train (3) by $B=B_{1}+k_{s} t_{p}$.

For $|\tau| \leq t_{p}$, the magnitude of the autocorrelation function of the train (3) can be written as the two-term product:

$$
\left|R_{u_{s}}(\tau)\right|=\left|R_{1}(\tau)\right| \cdot\left|R_{2}(\tau)\right| .
$$

The derivation of formula (4) for the case when $u_{1}(t)$ is an LFM pulse can be found in [1]. The same steps (see [1] for details) can be carried out for the general case with an arbitrary phase $\phi(t)$ which lead to the factoring formula (4). The first term in (4) is the magnitude of the autocorrelation function of any single subpulse in (3)

$$
\left|R_{1}(\tau)\right|=\left|\int_{-\infty}^{\infty} u(t) \overline{u(t-\tau)} d t\right|
$$

and the second term

$$
\left|R_{2}(\tau)\right|=\left|\frac{\sin (N \pi \tau \triangle f)}{N \sin (\pi \tau \triangle f)}\right|
$$

causes the appearance of the grating lobes at the points of the maxima of $\left|R_{2}(\tau)\right|$, i.e. at

$$
\tau_{n}=n \cdot \frac{1}{\triangle f}, \quad \text { where } n= \pm 1, \pm 2, \ldots,\left\lfloor t_{p} \triangle f\right\rfloor .
$$

To achieve a desired level of grating lobe suppression we require the values of $\left|R_{1}(\tau)\right|$ at these peaks to be less than some small value chosen a priori. We denote this value, which controls the level of suppression, by $\epsilon$. As the result, we come to the following set of inequalities

$$
\left|R_{1}\left(\tau_{n}\right)\right| \leq \epsilon
$$

which are discussed in the next sections.

\section{CASE 1: LFM PULSE TRAIN}

At this point we assume that $u_{1}(t)$ is a linear frequency modulated pulse

$$
u_{1}(t)=\frac{1}{\sqrt{t_{p}}} \operatorname{rect}\left(\frac{t}{t_{p}}\right) \exp \left(j \pi k t^{2}\right)
$$

with $k>0$. Note that the ultimate bandwidth of each pulse in the train (3) is $B=\left(k+k_{s}\right) t_{p}>0$.

Now the autocorrelation function for any single subpulse in train (3) is available in closed form

$$
\left|R_{1}(\tau)\right|=\left|\left(1-\frac{|\tau|}{t_{p}}\right) \operatorname{sinc}\left(B \tau\left(1-\frac{|\tau|}{t_{p}}\right)\right)\right|, \quad|\tau| \leq t_{p}
$$

which allows analysis of its shape. Consequently, some degree of control via the choice of the introduced parameters $\triangle f, B$ and $t_{p}$ is available.

It is easy to see that the set of inequalities (8) holds if

$$
\left|\sin \left(\pi B \tau_{n}\left(1-\frac{\tau_{n}}{t_{p}}\right)\right)\right| \leq \epsilon \pi B \tau_{n},
$$

for $n=1,2, \ldots\left\lceil t_{p} \triangle f\right\rceil-1$. Thus, if one seeks to suppress the grating lobes of the LFM train below the desired $\epsilon$-level, the values of $\triangle f, B$ and $t_{p}$ should be chosen such that the system (11) is satisfied.

We should remark at this point that although we have $\left\lceil t_{p} \triangle f\right\rceil-1$ inequalities in (11), some of them will be satisfied automatically for sufficiently large index $n$. Indeed, since the left-hand side of (11) does not exceed 1, the local maxima of $\left|R_{1}\left(\tau_{n}\right)\right|$ with $n=n_{*}+1, n_{*}+2, \ldots,\left\lceil t_{p} \triangle f\right\rceil-1$ and $n_{*}=\lfloor 1 /(\epsilon \pi(B / \triangle f))\rfloor$ are automatically less than the $\epsilon$-level and, hence, irrelevant for further discussion. Therefore, the number $n_{*}$ of relevant grating lobes depends on the desired level $\epsilon$ of suppression and the overlap ratio $B / \triangle f$ between pulses. Fig. 2 illustrates the behavior of the number $n_{*}$ of relevant grating lobes with respect to the overlap ratio $B / \triangle f$. Note that, if it is necessary to design the waveform with a small ratio $B / \triangle f$ and a small number of pulses $N$, then all (or almost all) the grating lobes should be taken into account. On the other hand, if a relatively large ratio is acceptable, one has to be concern only about the values of first few grating lobes regardless of the total number of pulses in the train.

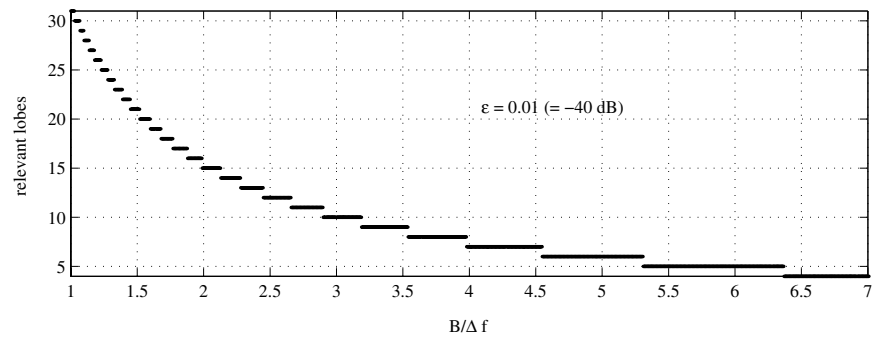

Figure 2: Dependence of the number $n_{*}$ of the potential grating lobes on the ratio $B / \triangle f$ for $\epsilon=0.01$.

Based on the above observation, we have to satisfy the system (11) only for $n=1,2, \ldots, n_{*}$. A pictorial representation for the solution of the system is given in Fig. 3, where 
the shaded regions correspond to the set of parameter values on which the inequalities (11) are satisfied. As one illustration of what can be produced by appropriate choice of points in the shaded regions of Fig. 3 we next present plots of the smallest ratios $B / \triangle f$ as a function of $t_{p} \triangle f$ and the corresponding values of $t_{p} B$ as a function of $t_{p} \triangle f$ (see Fig. 4) for $\epsilon=0.025$, and $N=11$. This choice of $\epsilon$ produces grating lobe level $\leq-30 \mathrm{~dB}$. It is evident from Fig. 4 that for large $t_{p} \triangle f$ it is possible to push the grating lobes below the desired level even if the ratio $B / \triangle f$ is quite small (we remind the reader that in the case of "Quick

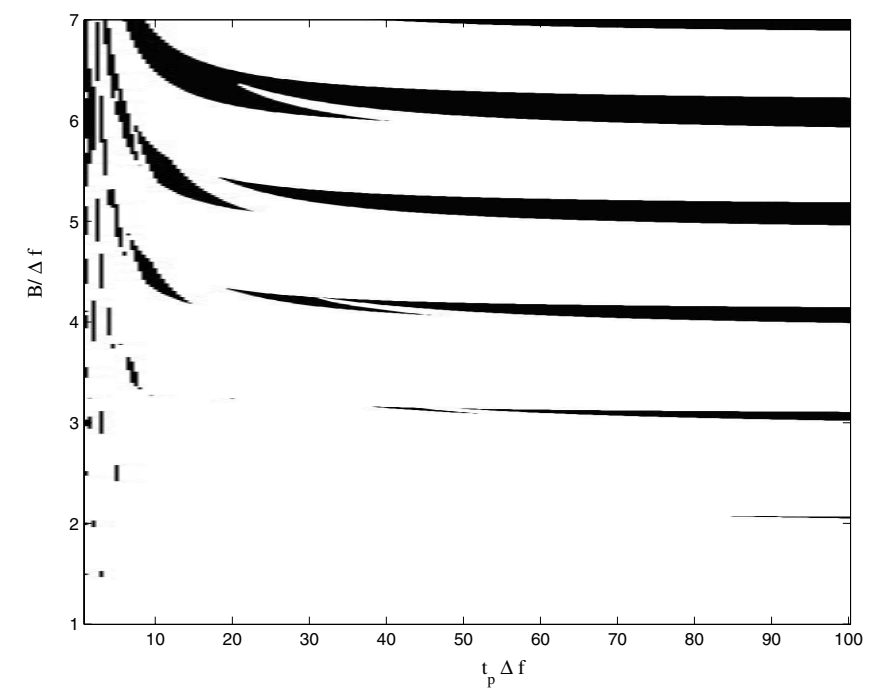

Figure 3: Shaded regions represent all possible values of $\left(t_{p} \triangle f, B / \triangle f\right) \in[1,100] \times[1,7]$ where (11) is satisfied.
Burst" the total number of pulses $N$ is supposed to be small). Thus, our approach to the grating lobes suppression problem allows us, compared to the approach presented in [1], to widen the range of the acceptable parameters significantly.

We note at this point that suitable selection of the points $\left(t_{p} \triangle f, B / \triangle f\right)$ in the shaded region of Fig. 3 can produce small values of $\left|R_{u_{s}}(\tau)\right|$ in substantial neighborhood of the $\tau_{n}$ 's, as opposed to just at the $\tau_{n}$ 's themselves. The choice of the parameter $\epsilon$ in (11) depends on an acceptable level of grating lobes, the number of pulses $N$ in the train, and the frequency step $\triangle f$.
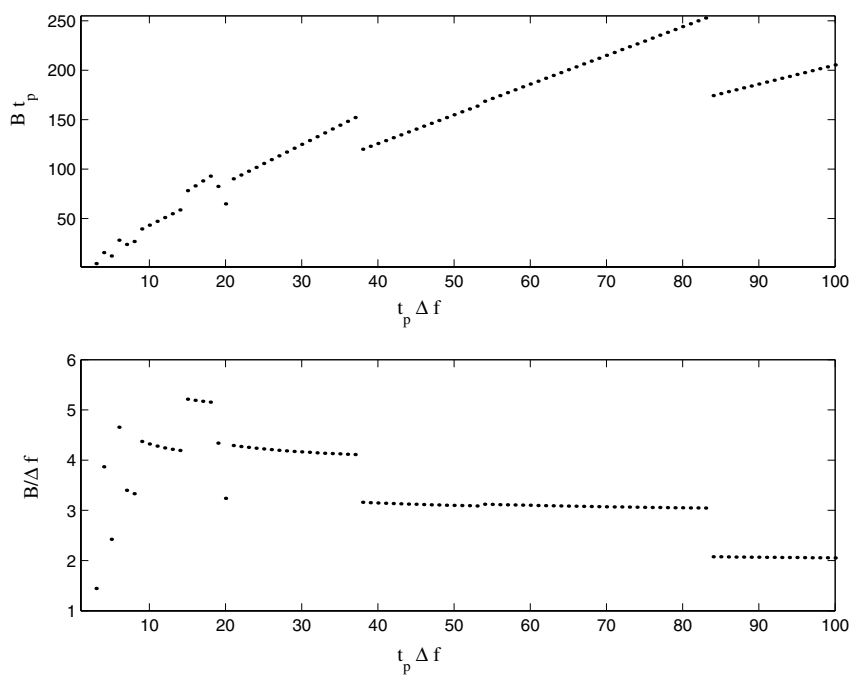

Figure 4: Relationship between $t_{p} B$ and $t_{p} \triangle f$ corresponding to the smallest possible ratio $B / \triangle f$ (top), smallest ratios $B / \triangle f$ for $t_{p} \triangle f=1,2, \ldots, 100$ (bottom).
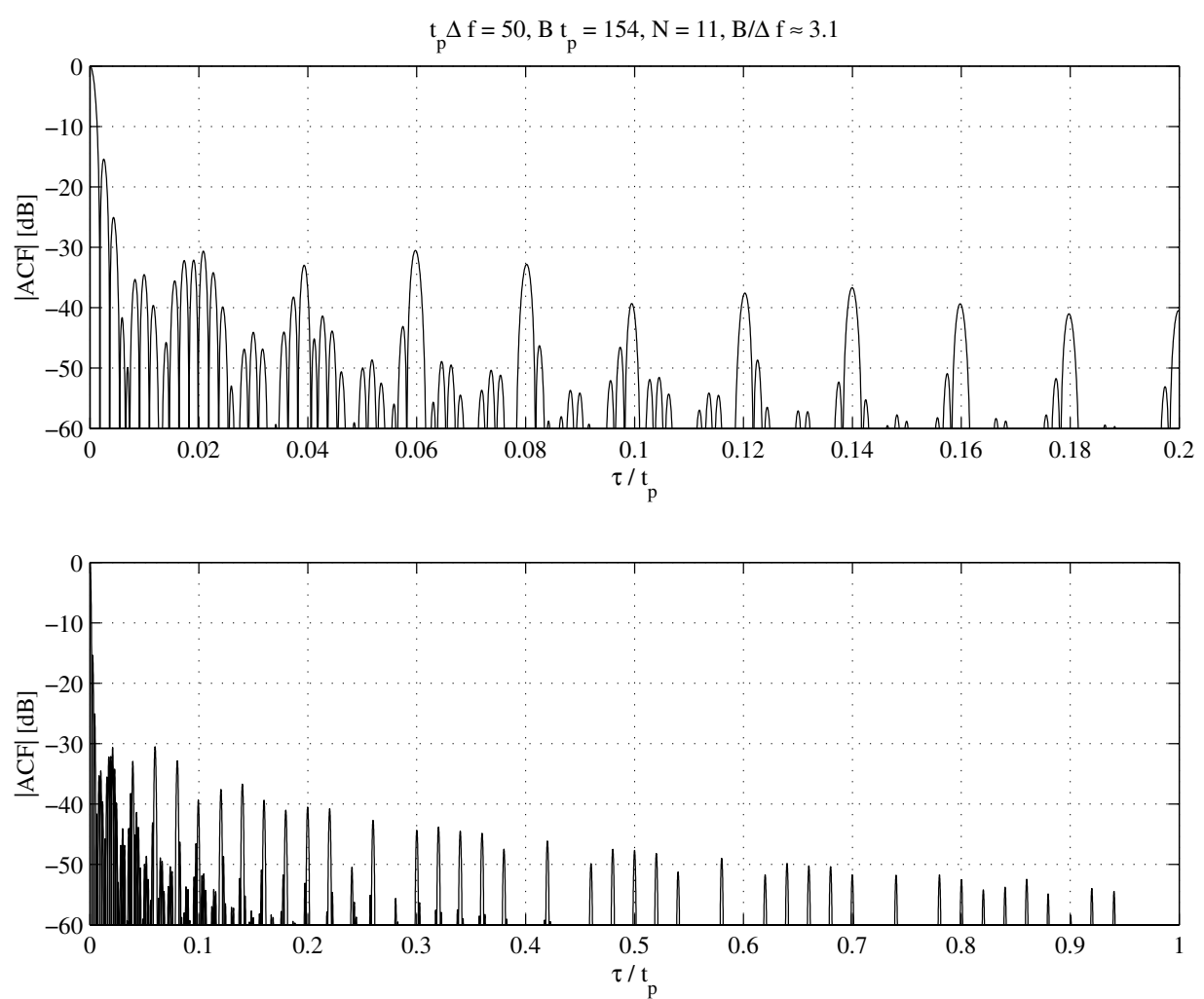

Figure 5: Partial autocorrelation function (in dB) for $t_{p} \triangle f=50, t_{p} B=154, N=11$ and $B / \triangle f \approx 3.1$ 
The magnitude of the autocorrelation function for some typical cases is shown in fig. 5-7. For each pair, the top drawing illustrates the $\left|R_{u_{s}}(\tau)\right|$ in the main lobe area and the bottom drawing displays $\left|R_{u_{s}}(\tau)\right|$ for $0 \leq \tau \leq t_{p}$. As is evident from the figures, all of the grating lobes are below the chosen level of $-30 \mathrm{~dB}$.
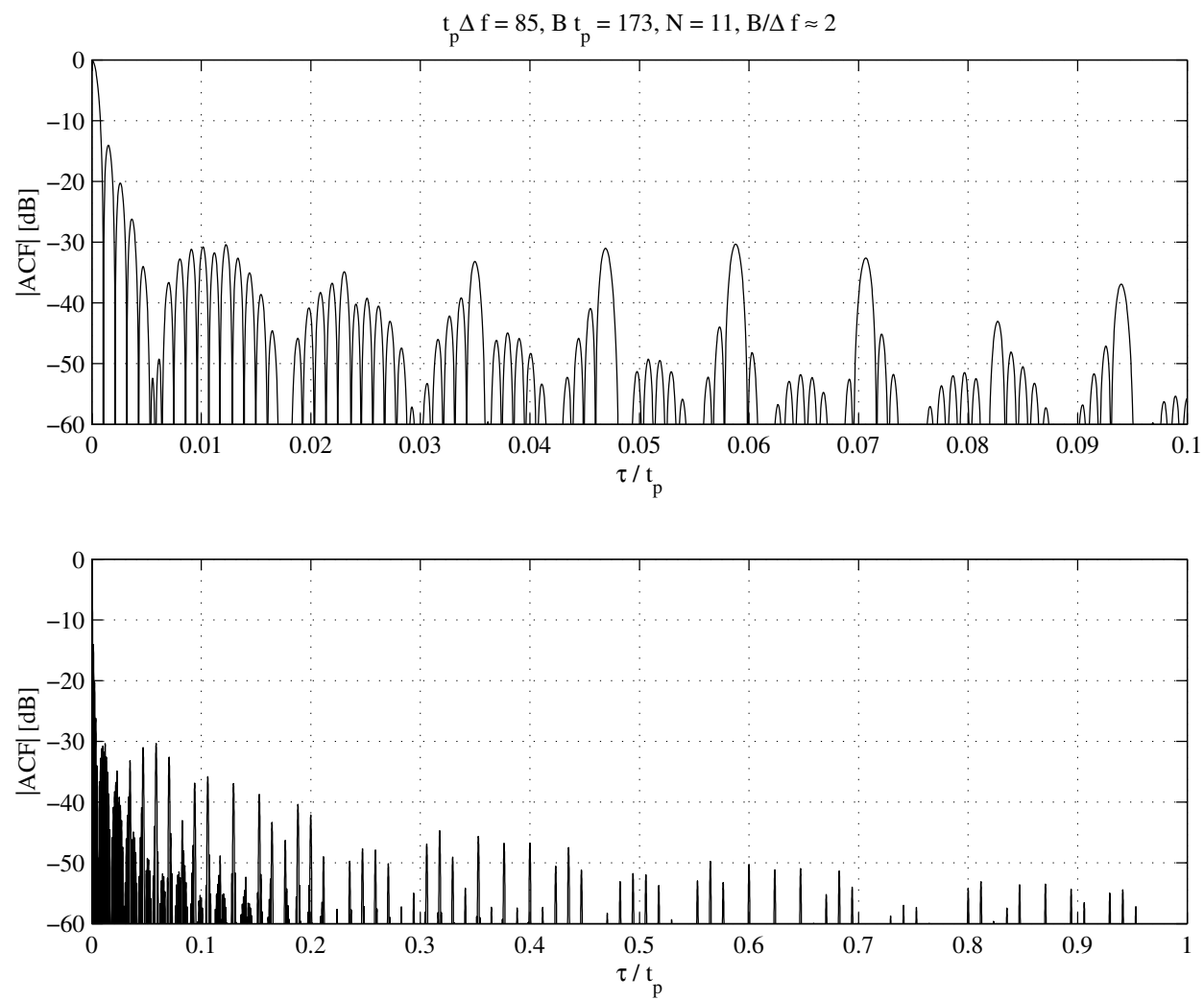

Figure 6: Partial autocorrelation function (in $\mathrm{dB}$ ) for $t_{p} \triangle f=85, t_{p} B=173, N=11$ and $B / \triangle f \approx 2$
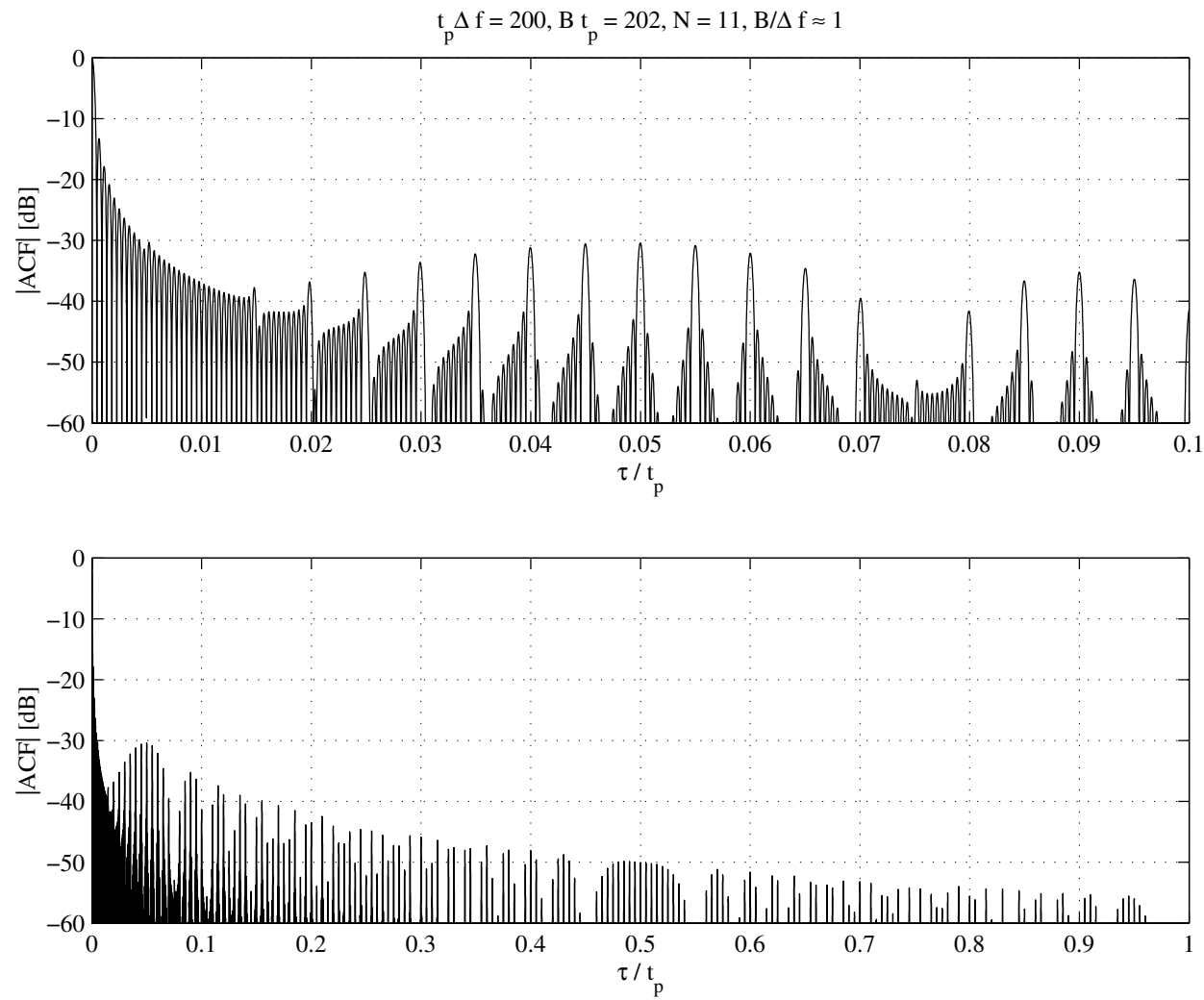

Figure 7: Partial autocorrelation function (in $\mathrm{dB}$ ) for $t_{p} \triangle f=200, t_{p} B=202, N=11$ and $B / \triangle f \approx 1$ 


\section{CASE 2: NON-LINEAR FM PULSE TRAIN}

Another issue that we would like to address in this paper is the high sidelobes in the close vicinity of the main lobe. Within the chosen construction of the stepped-frequency train with constant time duration $t_{p}$, constant bandwidth $B$, constant step $\triangle f$, and small ratio $B / \triangle f$, it is not possible to suppress these few sidelobes. Nevertheless, if a linear FM pulse is replaced by a non-linear FM pulse, then it is possible to suppress the sidelobes near the main lobe as well as the grating lobes. In this section we describe a numerical approach that allows a design of the phase $\phi(t)$ of such a non-linear FM waveform.

We consider $u_{1}(t)=1 / \sqrt{t_{p}} \cdot \operatorname{rect}\left(t / t_{p}\right) \exp (j \pi \phi(t))$ and impose an additional requirement of monotonicity on $\phi(t)$, since otherwise even small sufficiently numerous oscillations in $\phi(t)$ contribute large superfluous amounts of energy.

Our algorithm can be described as follows:

We take normalized $t: 0 \leq t \leq 1$ and assume $0 \leq$ $\phi(t) \leq B$. Then we subdivide $[0,1]$ into equal subintervals $0=t_{0}<t_{1}<\cdots<t_{N}=1$, where $t_{k}-t_{k-1}=\Delta t$. The corresponding phase increments are $\phi\left(t_{k}\right)-\phi\left(t_{k-1}\right)=h_{k}^{2}>0$. The $h_{k}^{2}$ can be thought of as providing a discretized version of the monotonic function $\phi(t)$. The use of the squared form of the $h_{k}$ 's in the algorithm automatically assures that phase increments are non-negative. We will think of the $h_{k}$ 's as parameters to be chosen in a way that provides an optimized shape of the autocorrelation function $R_{1}(\tau)$ associated to the phase $\phi(t)+k_{s} t^{2}$.

We next create a continuous version of a candidate phase function $\phi(t)$ by splining together the values $\left(t_{k}, \sum_{j=1}^{k} h_{j}^{2}\right)$. Now that we have our $\phi(t)$, depending on the parameters $\left\{h_{k}\right\}$, we compute the autocorrelation function $R_{1}(\tau)$ of the FM waveform $u(t)$ defined by the phase $\phi(t)+k_{s} t^{2}$ and the time support $t_{p}$, and then find

$$
\underset{\left\{h_{k}\right\}_{k=1}^{N}}{\arg \min }\left\|R_{1}(\tau)\right\|_{S}^{2}, \quad \text { subject to } \quad \sum_{k=1}^{N} h_{k}^{2}=B,
$$

where

$$
S=\bigcup_{n \in I}\left[\frac{n}{\triangle f}-\delta, \frac{n}{\triangle f}+\delta\right] \bigcup\left[\frac{3}{2 \triangle f N}-\delta, \frac{3}{2 \triangle f N}+\delta\right]
$$

and $I=\left\{ \pm 1, \pm 2, \ldots,\left\lfloor t_{p} \triangle f\right\rfloor\right\}$.

The choice of the set $S$ depends on the parameter $\delta$ that determines the width of the intervals where the values of the autocorrelation function $R_{u}(\tau)$ are desired to be small, i.e. the neighborhoods of the peaks of $\left|R_{2}\right|$ as well as the neighborhood of the first local maximum of $\left|R_{2}\right|$ located at $\frac{3}{2} \cdot \frac{1}{\triangle f N}$.

The steps of the described algorithm are carried out numerically using well-known standard techniques of optimization, and give good results, illustration of which are given in figures below.

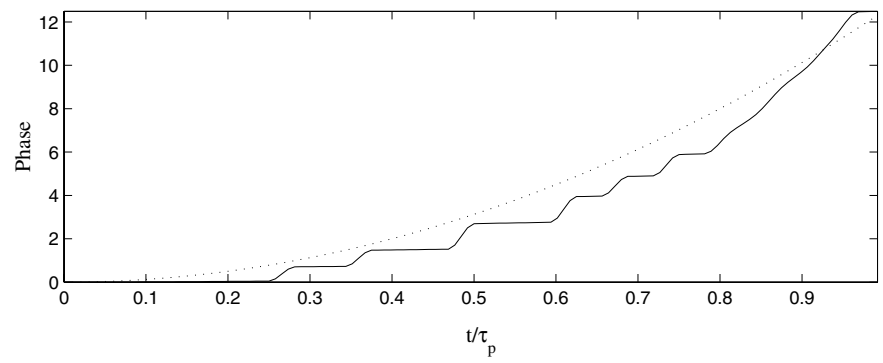

Figure 8: Phase of the non-linear FM pulse (solid) and linear FM pulse (dashed)
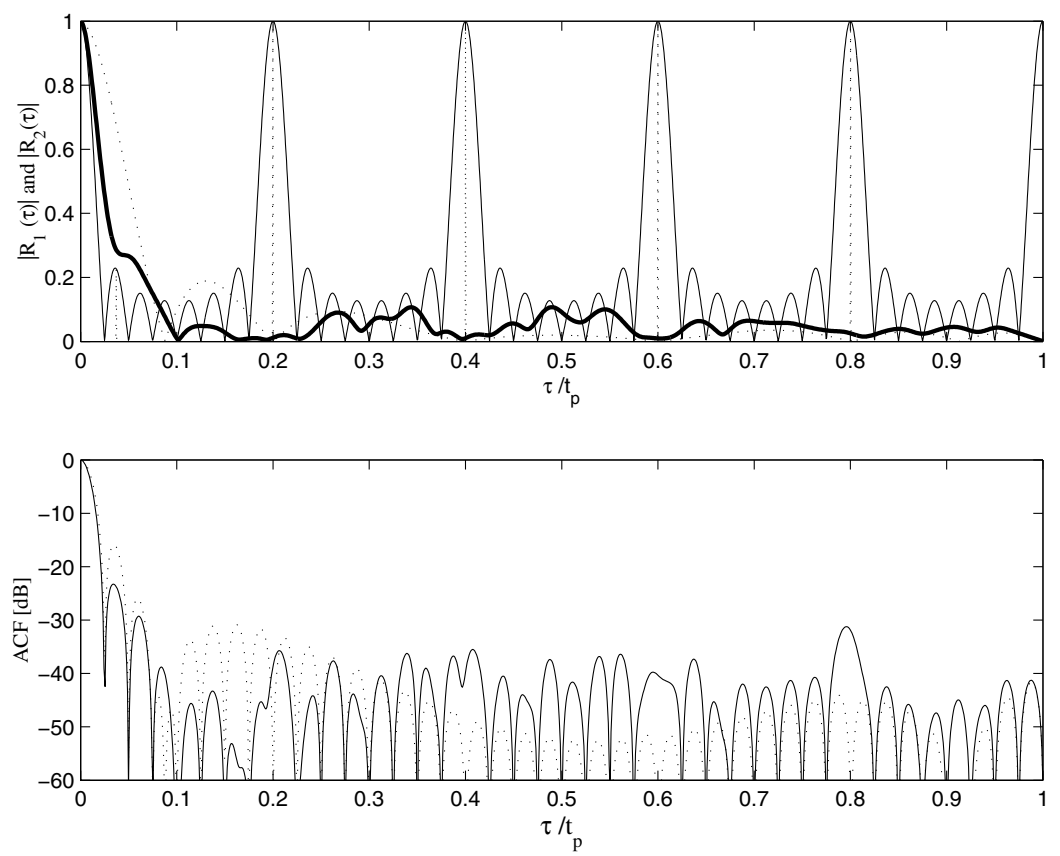

Figure 9: Stepped frequency train with suppressed grating lobes. Top: $\left|R_{2}(\tau)\right|$ (solid), $\left|R_{1}(\tau)\right|$ corresponding to the linear FM pulse (dotted), $\left|R_{1}(\tau)\right|$ corresponding to the non-linear FM pulse (thick solid). Bottom: Partial autocorrelation function in logarithmic scale of stepped-frequency train based on LFM (dotted) and NLFM (solid) pulses. 

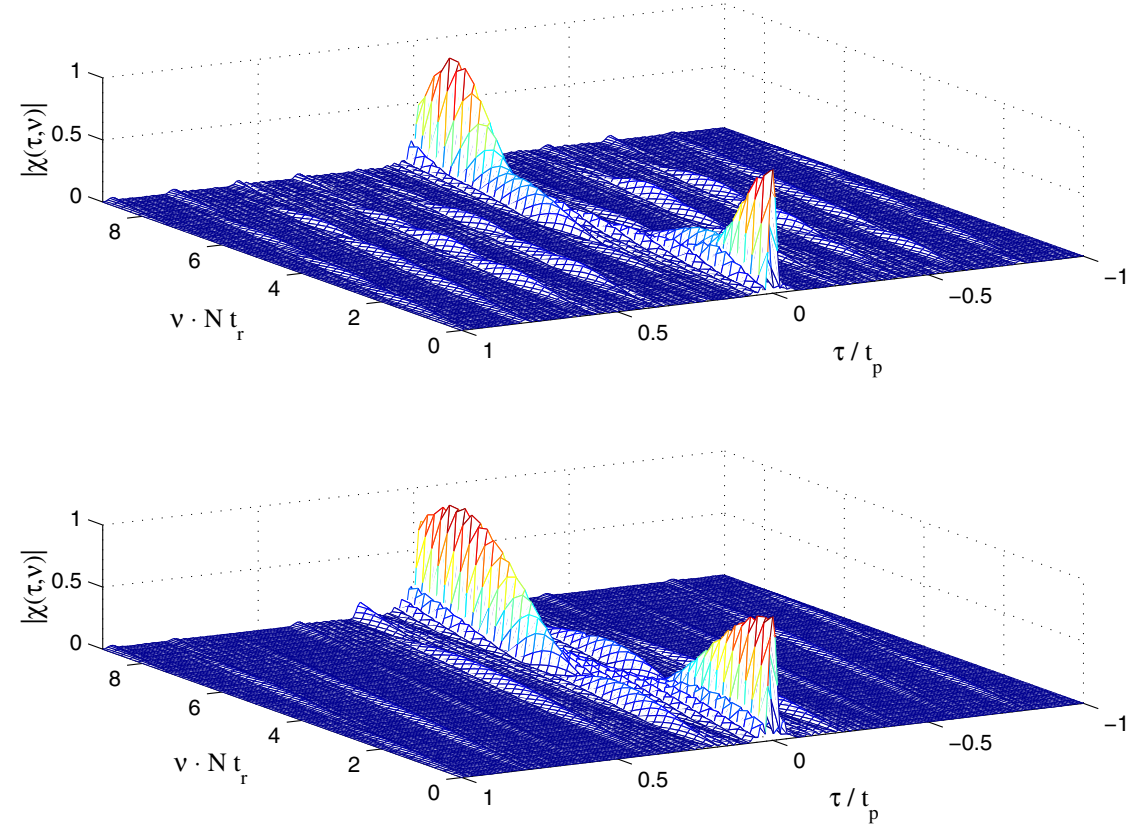

Figure 10: Ambiguity function of stepped-frequency train of LFM pulses (bottom) and NLFM pulses (top) with suppressed grating lobes.

Fig. 8 shows the phase $\phi(t)$ of the non-linear FM (solid line) that was obtained via the described algorithm and the phase of the linear FM pulse (dashed line) $u_{1}(\tau)$ with $k=$ 11.75. The phase $\phi(t)$ was designed so that the main lobe of $\left|R_{u}(\tau)\right|$ has almost the same width as one arch of $\left|R_{2}(t)\right|$, and so that the grating lobes of $\left|R_{u_{s}}(\tau)\right|$ are suppressed (see Fig. 9 (top)). The location of the set $S$ and the corresponding values of $\left|R_{2}(\tau)\right|$ for $\tau \in S$ are indicated on figure 9 by dotted line. The thick solid line on figure 9 corresponds to the autocorrelation $\left|R_{u}\right|$ and the dashed line corresponds to the autocorrelation $\left|R_{1}\right|$ of the chirp pulse $u_{1}(\tau)$. Figure 9 (bottom) illustrates part of the autocorrelation function (with delay axis limited to $\left|\tau_{p}\right| \leq t_{p}$ ) of the stepped frequency train in a logarithmic scale based on the non-linear frequency modulated pulse (solid line) and on the chirp pulse in the logarithmic scale. The hight of the first sidelobe corresponding non-linear FM is lower that corresponding to LFM by $7 \mathrm{~dB}$.

\section{CONCLUSION}

Frequency stepping is one of the known techniques employed in modern radar to achieve high range resolution by combining a sequence of narrowband pulses that span the desired bandwidth. The downside of such a construction is the possible presence of high grating lobes. In this paper we have presented an approach which allows us to suppress all of the grating lobes below a desired threshold level. We have discussed the relationship between the parameters determining the stepped frequency waveform and demonstrated how they should be chosen in order to obtain small grating lobes. We have also discussed the problem of high sidelobes in the vicinity of the main lobe, and have shown that it is possible to suppress these, as well as the grating lobes, if a linear FM pulse is replaced by a non-linear FM pulse. We have described a numerical approach to the design of the phase of such a non-linear FM waveform.

\section{ACKNOWLEDGMENTS}

This work is being sponsored in part by the U.S. Army Research Laboratory and the U.S. Army Research Office under Contract DAAD19-03-1-0329.

\section{REFERENCES}

1. N. Levanon, E. Mozeson, "Nullifying ACF Grating Lobes in Stepped-Frequency Train of LFM pulses", IEEE Trans. on AES, vol.39, (April) 2003, p. 694-703.

2. D.J. Rabideau, "Nonlinear synthetic wideband waveforms", Proc. 2002 IEEE Radar Conf., (May) 2002, p. 212-219.

3. P. M. Woodward, Probability and Information Theory, with Applications to Radar, Pergamon Press, 1953.

4. M. I. Skolnik, Introduction to Radar Systems, McGraw Hill, 1980.

Irina Gladkova received a B.Sc. degree in mathematics from Donetsk State University, Ukraine, in 1989, a Ph.D. in physi$\mathrm{cal} /$ mathematical sciences from the Institute of Applied Mathematics and Mechanics of the National Academy of Sciences of Ukraine in 1993, and a Ph.D. in Mathematics from The Graduate Center of the City University of New York in 1998.

She has been a faculty member of City College of New York since 1998, where she is an Assistant Professor in the Department of Computer Science. Her areas of interests are numerical methods, radar waveform design, and data compression.

Department of Computer Science, City College of New York, 138th Street at Convent Ave, New York, NY 10031 